\title{
IDEAS DEL INFINITO, PERCEPCIONES Y CONEXIONES EN DISTINTOS CONTEXTOS: EL CASO DE ESTUDIANTES CON CONOCIMIENTOS PREVIOS DE CÁLCULO
}

\author{
Garbin D., Sabrina \\ Universidad Simón Bolivar
}

\begin{abstract}
Resumen. En este artículo presentamos una investigación que surge de un especial interés por estudiar y explorar el caso del infinito, en su dualidad potencial-actual, en un nivel donde ya se han introducido conceptos formales del cálculo diferencial e integral, y donde empiezan a aparecer interconexiones y confusiones entre la «imagen formal» e «imagen informal» de estos conceptos. El estudio pretende contribuir con el debate de la problemática del infinito e infinitos a nivel universitario. Se enmarca en un estudio cualitativo; el análisis de datos es inductivo y el foco de investigación es de carácter exploratorio, descriptivo e interpretativo. Participaron en el estudio 89 estudiantes con edades comprendidas entre 17 y 25 años.
\end{abstract}

Palabras clave. Infinito, esquemas conceptuales, percepciones, imagen formal e informal, cálculo.

Summary. We present a research springing from a special interest in studying and exploring the infinite and its duality power-present, at a level where formal concepts of differential and integral calculus have already been introduced and where there appear interconnections and confusion between the «formal image» and the «informal image» of these concepts. This paper attempts to contribute to the debate of the finite and infinite problems at university level. It is framed within a qualitative study, the analysis of date is inductive and the focus of the research is of an exploratory, descriptive and interpretative kind. Eightynine students aged between 17 and 25 took part in this study.

Keywords. Infinite, conceptual schema, perceptions, formal and informal image, calculus.

\section{INTRODUCCIÓN}

El interés por el infinito o los infinitos matemáticos, en la matemática y en otras disciplinas como la filosofía o la historia, es un asunto milenario. En las últimas décadas, la problemática del concepto de infinito matemático también lo es para la psicología y para la didáctica de las matemáticas.

Son muchas las investigaciones realizadas, trabajos que representan interés histórico, epistemológico, psicológico, filosófico o didáctico. D’Amore $(1996,1997)$ recoge numerosos títulos (280) del año 1951 al 1996 y muestra con éstos la dirección que han ido tomando las investigaciones. Después del 1996 siguen apareciendo nuevos trabajos, pero más recientemente la revista Educational Studies in Mathematics dedica, al tema del infinito, el volumen núm. 48 (2001). Tall y Tirosh afirman que esta selección de artículos pretende contribuir, desde la psicología del pensamiento matemático, al debate que, a lo largo de la historia, se ha generado sobre el concepto y su desarrollo a lo largo del tiempo, superando obstáculos tanto intuitivos como formales o psicológicos. Se estudia en estos artículos el infinito distinguiéndolo entre los infinitos y los infinitesimales del cálculo y los infinitos cardinales introducidos por Cantor. Se comienza con su desarrollo histórico y se consideran las distintas maneras en que se extrapola la experiencia finita a las varias teorías del infinito. Se sigue con la presentación 
de estudios empíricos sobre las concepciones de los estudiantes y sobre la enseñanza de los infinitos en diferentes contextos. En particular se aborda la enseñanza de los límites en el cálculo, específicamente de sucesiones, y del infinito cardinal en la matemática formal.

Por otra parte, Garbin y Azcárate $(2000,2002)$ y Garbin (2000) se hacen una serie de preguntas que derivan de plantear el problema de estudio. El interrogante consiste en cuál es la posible influencia de los lenguajes matemáticos en la concepción del infinito actual y en las inconsistencias de los alumnos. La primera experiencia de la investigación llevó a querer identificar las inconsistencias y representar, categorizar y analizar las situaciones de coherencias que manifiestan los alumnos en relación con sus esquemas conceptuales asociados al concepto de infinito actual y que se contextualizan en problemas expresados en lenguajes matemáticos diferentes: verbal, geométrico, gráfico, algebraico y analítico. Estos estudios se realizaron con estudiantes entre 16-17 años.

Trabajar en el ámbito universitario hace surgir un especial interés por estudiar y explorar el caso del infinito, en su dualidad potencial-actual, en especial el nivel donde ya se empezaron a introducir conceptos formales del cálculo diferencial e integral, y donde empiezan a aparecer interconexiones y confusiones entre la «imagen formal e informal» (Tall, 2001) de estos conceptos. Este artículo pretende contribuir con el debate de la problemática del infinito e infinitos a nivel universitario, y profundiza y completa lo presentado en Garbin (2003).

En particular, el estudio pretende: $a$ ) analizar los efectos que tiene en la percepción de los estudiantes universitarios, con conocimientos previos de cálculo diferencial e integral, la presencia implícita del «infinito pequeño» en preguntas planteadas en distintos contextos matemáticos, a partir de los esquemas conceptuales de los alumnos; $b$ ) estudiar qué tipo de conexiones y qué focos de atención presentan y establecen en las distintas cuestiones en que está presente el «infinito pequeño», así como establecer una posible relación entre los focos de atención y conexiones y la percepción del infinito que éstos puedan establecer.

\section{ANTECEDENTES Y FUNDAMENTACIÓN TEÓ- RICA}

En la introducción se afirma que son numerosas las investigaciones en didáctica sobre el infinito matemático pero algunas se pueden considerar como pilotos y pilares en el área del infinito matemático. Fischbein (1978), Fischbein, Tirosh y Hess (1979), Tirosh (1991), Nuñez (1994), Moreno y Waldegg (1991), Tsamir y Tirosh $(1995,1999)$ revelan entre otras cosas que la intuición natural del infinito es sumamente inestable y que las situaciones de conflicto que se presentan dependen de las influencias conjeturales y contextuales.

De especial interés para nuestro estudio son algunos resultados y aspectos de los trabajos de Fischbein (1978), Fischbein, Tirosh y Hess (1979), Nuñez (1994), Fischbein (2001), Kidron (2002), Garbin (2000), Garbin y
Azcárate (2002) y Garbin (2003), que a continuación se reseñan. Ante estos hallazgos resulta normal preguntarse qué pasa con estudiantes de niveles superiores que ya han tenido contacto con conceptos formales de cálculo diferencial e integral, interrogante que sustenta el problema de investigación que aquí se presenta.

Fischbein reveló la naturaleza conflictiva de las intuiciones del infinito en los estudiantes, enfocando su investigación en el infinito potencial como un proceso de límite y el infinito actual en la teoría de números cardinales. Fischbein (1978) encontró, en estudiantes de $8^{\circ}$ y $9^{\circ}$ grado, que la concepción intuitiva del proceso de límite tiende más a ser un proceso infinito que un valor finito de un límite. Esto fue confirmado más tarde (Fischbein, Tirosh y Hess, 1979; Fischbein, Tirosh y Melamed, 1981; Garbin y Azcárate, 2000, 2002).

Uno de los interrogantes de nuestro cuestionario involucra la serie $1 / 2+1 / 4+1 / 8+\ldots$ El estudio de esta serie como la representación de la suma de los segmentos, resultado de divisiones sucesivas de un segmento $A B$ de longitud 1 en mitades, tuvo interés para estos autores. Matemáticamente esta serie (convencionalmente) es «igual» a 1; sin embargo, «psicológicamente» esta serie «tiende» a 1 (Fischbein, 2001). En el estudio de Fischbein, Tirosh y Melamed (1981), los estudiantes contestaron que dicha suma es: infinita, tiende a 1 y es igual a 1 (con un número pequeño de estudiantes que afirmaron que es igual a 1). Este resultado fue igualmente encontrado en Garbin (2000), Garbin y Azcárate (2002), pero además de las respuestas anteriores hubo una repuesta adicional, algunos alumnos dieron un valor a la suma tomando ésta como una suma finita, es decir, evadieron la infinitud de la suma. Los esquemas finitos prevalecieron en algunos estudiantes a pesar de la representación infinita de la suma.

Kidron (2002) propuso un estudio para investigar la habilidad de los estudiantes de high school (grade 11, $\mathrm{N}=63$ ) de percibir la suma como un límite, como un objeto. Los estudiantes miraron el concepto desde dos diferentes acercamientos y trabajando en un laboratorio equipado con computadoras con el software Mathematica. En el estudio se encontró la misma percepción de suma infinita como en otros estudios; sin embargo, el $43 \%$ expresó una percepción de la suma infinita como un límite y el $56 \%$ escribió $1+1 / 2+1 / 4+\ldots=2$, un porcentaje «balanceado» en ver la suma como proceso y como concepto.

Nuñez (1994) hace uso de la primera paradoja de Zenón, de la división para ofrecer un acercamiento a cómo la mente construye la idea de infinito; y lo hace con alumnos de edades entre 8, 10, 12 y 14 años. Nuñez afirma que la situación de la paradoja de Zenón presenta una situación de subdivisiones, un infinito pequeño, que implica una coordinación simultánea entre el creciente número de pasos y el decreciente resultado parcial que debe ser operado. El concepto de suma infinita tiene dos «tipos» de iteraciones («divergente»y «convergente»), y diferente «naturaleza» del contenido («cardinalidad» y «espacio»). Estos procesos explicitados por Nuñez fueron tomados en cuenta para construir las líneas de coherencia, instrumento de análisis que se presenta en la metodología, dado que, en el concep- 
to involucrado en las cinco preguntas del cuestionario, están presentes los dos «tipos» de iteraciones y la diferente «naturaleza» del contenido.

Los estudios realizados con estudiantes de 16-17 años (Garbin, 2000; Garbin y Azcárate, 2000, 2002) no sólo son antecedentes, sino que, como en éstos, el tema de estudio, la naturaleza del problema y el propósito de la investigación, entre otros, ofrecen la perspectiva teórica del trabajo que se está presentando en este artículo, la cual, en cuanto al concepto de infinito, ya se ha reflejado con los antecedentes.

El estudio se enmarca en el dominio del llamado pensamiento matemático avanzado, teoría cognitiva desarrollada por Tall y Dreyfus. Los conceptos de esta teoría que se tratan son: a) de manera particular, el concept image que llamamos esquema conceptual y entendemos como: «el conjunto de todas las imágenes mentales del estudiante asociadas al concepto, juntamente con todas las propiedades que le caracterizan» (Tall y Vinner, 1981); informal image y formal image (Tall, 2001), que nombramos imagen informal y formal, la primera es algún tipo de imagen que se tiene antes del acercamiento con teorías axiomáticas, y la segunda con palabras de Tall consiste en la parte del esquema conceptual que es formalmente deducido de los axiomas. Esto enfoca el problema específico de la investigación, la tensión entre el infinito «perceptual» y el infinito «formalizado».

Por el tipo de preguntas que se plantea a los estudiantes en este estudio y por el nivel de los alumnos en que no ha sido formalizada la teoría de la cardinalidad de Cantor resulta imprescindible considerar el tema de la intuición, su rol, características y clasificación (Fischbein, 1998) También es importante considerarlo, por ser el conocimiento intuitivo un camino básico, junto al analítico, en la actividad matemática, como mostró Fischbein en su artículo «Intuition and Proof» (1982).

\section{METODOLOGÍA}

La investigación se enmarca en un estudio de tipo cualitativo. El análisis de datos es inductivo, ya que las interpretaciones y categorías se construyen a partir de la información obtenida. Se sigue, en parte, la metodología adoptada en Garbin (2000) y Garbin y Azcárate (2002).

\section{Instrumentos de recogida de datos y descripción de los participantes}

La experiencia se realiza con 89 estudiantes universitarios con edades comprendidas entre 17 y 25 años (73 de los 89 tienen entre 18 y 20 años). Nueve estudiantes de ciencias (química y física) y 80 estudiantes de ingeniería (polímeros, mecánica, metalmecánica, computación, eléctrica, producción, electrónica, geofísica y metalúrgica). Los alumnos pertenecían a tres secciones de matemáticas V, formadas aleatoriamente por el Departamento de Matemáticas. El desempeño de los estudiantes es variado, el grupo no tenía características específicas resaltables salvo el tipo de carrera a la cual pertenen. Los estudiantes tienen conocimientos previos formales sobre límites, derivadas, integrales, sucesiones y series; todos estudiaron los mismos cursos básicos (Matemáticas I, II, III y IV) dictados por el Departamento de Matemáticas de la Universidad Simón Bolívar, de Venezuela.

Dos cuestionarios fueron aplicados a los estudiantes: $\mathrm{C}_{1} \mathrm{y}$ $\mathrm{C}_{2}$. El primero consta de cinco preguntas, mientras que el segundo sólo presenta una cuestión (problemas considerados en Garbin, 2000; Garbin y Azcárate, 2002). Las cinco preguntas del $\mathrm{C}_{1}$ (Anexo I) están inspiradas en la primera paradoja de la división, de Zenón, y diferenciadas por el contexto.

La paradoja de la dicotomía pone en superficie dos aspectos. Si nos queremos mover del punto 1 al 2 de la recta real, podemos hacerlo con un procedimiento finito, nos movemos toda la unidad de una vez; o podemos utilizar un procedimiento infinito, moviéndonos $1 / 2$ unidad, después $1 / 4$ unidad, y así sucesivamente. Si se ignora la posibilidad de que hay puntos distintos a una distancia infinitesimal entre uno y otro, es evidente que ambos procedimientos conducen al mismo punto. Este hecho está expresado en la «igualdad» $1+1=1+1 / 2+1 / 4+1 / 8+\ldots$ que Zenón consideraba paradójica. El hecho es que asumía a priori que no existía el «infinito actual»y, entonces, que ningún proceso infinito podría considerarse completo (Rucker, 1995, p. 117).

En particular, las preguntas presentan una situación que, en lenguaje de Nuñez, (1994) presenta un infinito pequeño que implica cognitivamente una coordinación simultánea, el creciente número de divisiones y el decreciente «espacio» que se recorre o «resultado parcial» que se opera.

Para el segundo cuestionario $\left(\mathrm{C}_{2}\right)$ (Anexo I) se seleccionaron tres (1, 3 y 4) de las cinco preguntas del $C_{1}$ y se aplicaron al mismo grupo de alumnos después de aplicar el $C_{1}$. Su objetivo es extraer información sobre qué tipo de conexiones y focos de atención presentan y establecen los estudiantes a partir de las preguntas 1,3 y 4 del $\mathrm{C}_{1}$.

\section{METOLOGÍAS ESPECÍFICAS Y ANÁLISIS}

\section{Redes sistémicas y esquemas conceptuales}

Se opta por el uso de las redes sistémicas (Bliss, Monk y Ogborn, 1983) como sistema de clasificación y representación de los datos cualitativos obtenidos a partir de las respuestas dadas por los alumnos en el cuestionario $\mathrm{C}_{1}$. Se elabora una red sistémica para cada una de las preguntas del $C_{1}$. En el gráfico 1 se puede observar a modo de ejemplo la red sistémica de la pregunta 4 y en los anexos se observan el resto de las redes sistémicas elaboradas. Éstas consisten en una determinada configuración de los datos, que permite mirar de manera efectiva todas las respuestas de los alumnos encuestados. Como se puede observar, las redes se estructuran en forma de árbol con ramas que se subdividen en «clases» (se usa como formalismo la barra (I)), que son categorías que se excluyen entre ellas. Los estudiantes habían sido identificados aleatoriamente con números del 1 al 89. 
Podrías determinar el valor de la función? (VF)

\begin{tabular}{l|l} 
Tiende (se acerca, se aproxima) a 0 & $\begin{array}{l}\lim _{n \rightarrow \infty} f(x)=0 \\
(1)(3)(5)(6)(7)(8)(17)(18) \\
(21)(24)(28)(29)(30)(31)(45)(47) \\
(48)(50)(53)(55)(57)(59)(62)(69)(71)(84)(86)\end{array}$
\end{tabular}

Se va convirtiendo en una línea recta

Será el eje de las abscisas

$$
x \longrightarrow \infty, y \longrightarrow \infty \text {, }
$$

Se acerca a 1

$(40)$

\begin{tabular}{l|l} 
Tiende (se acerca, se aproxima) a 2 & $\begin{array}{l}\lim _{x \rightarrow \infty} f(x)=2, \text { pero nunca será } 2 \\
(2)(10)(12)(16)(19)(20)(23)(25)(26)(27)(33)(35)(38)(39)(44)(56) \\
(59)(60)(63)(70)(76)(77)(79)(85)(88)\end{array}$
\end{tabular}

$$
\text { En el } \infty, f(x)=2 \quad(14)(46)(72)(75)
$$

Por comodidad, la oscilación va a variar muy poco

$V F$

El valor de $f(x)$ se hace cada vez más pequeño

El valor es 2 Tiende a transformarse en una recta

Cuando se hace extremadamente grande

$(9)(32)(34)(37)(43)(68)(82)(83)$

Se podrá calcular considerando una función trigonométrica y cuyo valor tienda a $\infty$

$\lim _{x \rightarrow \infty} f(x)=2 \quad(36)(81)$

$\lim _{n \rightarrow \infty} \frac{a \cos x}{b^{n}}=0$

Es infinita

$(67)$

Tiende a $\infty$

$(61)$

\begin{tabular}{l|ll} 
No se puede determinar el valor & $f(x) \rightarrow 0$
\end{tabular}

No contesta $\mid(41)(58)(65)(66)(74)(78)$ 
A continuación se describe, por pregunta, la clasificación que ha permitido realizar las redes sistémicas, y se presentan unas primeras conclusiones. No se describe la pregunta 5, dado que su similitud con la pregunta 3 no aporta datos nuevos para esta primera parte, pero sí se tendrá en cuenta para las clasificaciones que seguirán.

\section{Pregunta 1}

a) El proceso infinito no es completo.

En esta categoría, la concepción del infinito es potencial. El punto de bisección no coincide con el punto B: $a$ ) 39 alumnos afirman que el punto de bisección se aproxima y tiende al punto extremo B. Veinte alumnos de éstos afirman, además, que siempre hay una distancia para biseccionar y 3 , que en una recta o segmento hay infinitos puntos. $b$ ) Dos estudiantes fundamentan su respuesta con el uso de límites, afirmando que el límite de la sucesión $1 / 2^{n}$ tiende a $0 . c$ ) Dos estudiantes fundamentan relacionando la situación del segmento con la serie $\Sigma 1 / 2^{n}$ Para uno de ellos la serie nunca es 1; para el otro, la serie no converge.

\section{b) El proceso infinito es completo.}

Para este grupo de estudiantes, el punto de bisección coincide con el punto extremo B. La situación es percibida como actual. Entendemos, en este artículo, el infinito actual, como el que está asociado a la idea de totalidad, de completes y de unidad. Un proceso (potencialmente infinito en sus orígenes) se considera acabado y los límites alcanzados.

- Doce estudiantes argumentan que el punto de bisección alcanza al punto extremo B porque las bisecciones son infinitas. Los matices son distintos:

- El límite de la sucesión $1 / 2^{n}$ tiende a 0 cuando $n$ tiende a infinito ( 2 estudiantes).

- Afirma: «[...] es como una sucesión de puntos que converge a $B »$ (1 estudiante).

- Argumentan que es un caso límite, o usan límites o series (2 estudiantes).

- Argumenta geométricamente: «El esquema representa la razón del segmento correspondiente entre un número cada vez más grande» (1 estudiante).

- Un estudiante explicita, en su argumento, la «divergencia» $\mathrm{y}$ «convergencia» del proceso en el sentido de Nuñez (1994): «[...] cuando la cantidad de divisiones tiende a infinito el tamaño del segmento tiende a 0 y alcanza al punto $B »$.

- Dos estudiantes acuden a lo «visual» y a la «cercanía»: «Estará tan cerca de B que no se va a distinguir el punto de bisección y el punto $B »$.

- El punto B es alcanzado por la finitud del segmento AB (1 estudiante).
Un estudiante que no contesta la pregunta ni afirmativa ni negativamente refleja su concepción de la situación: «[...] yo puedo decir que el segmento $A B$ es la suma de cada una de las bisecciones, $A B=\Sigma 1 / 2^{n}$, suma desde $n=1$ a infinito». Para este alumno, dicha igualdad presupone una división actual del segmento AB.

c) Que el proceso infinito sea completo o no depende del aspecto que se atiende.

En esta categoría se vislumbra la situación paradójica de la pregunta. Un alumno afirma que, por la infinitud, matemáticamente es imposible, es decir, se sitúa ante un infinito potencial pero visualmente afirma que sí es posible. Otro afirma que «idealmente» sí es posible que el proceso infinito sea completo. Interpretamos que a nivel de ideas la percepción puede ser actual, pero afirma que en la «realidad» siempre falta un trozo por recorrer.

d) El proceso es finito, el punto B es alcanzado.

Un solo estudiante explicita que se tiende de manera finita al punto extremo B y, por esta razón, el punto B es alcanzado. Es decir, la finitud del segmento determina la finitud de las bisecciones.

e) El proceso infinito de bisección no determina la respuesta.

En esta categoría (13 alumnos) no es la concepción o intuición del infinito potencial o actual la que determina la respuesta. La idea está en que el punto B, por ser extremo, no puede ser un punto de bisección, lo cual contradice el hecho de pensar que el punto de bisección coincida con el punto B.

f) Conceptos asociados.

No todos los alumnos nombran en sus argumentos los conceptos de límite, sucesiones, o suma infinita. En la siguiente tabla encontramos el número de alumnos que hacen referencia, en sus respuestas, a los conceptos de límite, sucesiones, series y la palabra tendencia. Dos alumnos para negar la posibilidad de que el punto de bisección alcance al punto extremo $\mathrm{B}$ hacen referencia a la paradoja de Zenón.

\begin{tabular}{|c|c|c|c|c|}
\hline & Límite & $\begin{array}{c}\text { Sucesiones } \\
\text { y series }\end{array}$ & $\begin{array}{c}\text { Límites } \\
\text { y series }\end{array}$ & Tendencia \\
\hline $\begin{array}{c}\text { Núm. de } \\
\text { alumnos }\end{array}$ & 13 & 7 & 2 & 4 \\
\hline
\end{tabular}

\section{Pregunta 3}

a) El proceso infinito no es completo, la serie converge.

Treinta y dos alumnos afirman la convergencia de la serie pero sin aceptar que se alcance al punto límite. La convergencia ha sido considerada hacia el 1,2 o a un número sin indicar el valor específico. Las razones han sido variadas como se puede observar en la figura del 
anexo IV; sin embargo, ninguno demuestra formalmente la convergencia de la serie. Sobresale la idea de que se va aproximando al valor de convergencia y que no llega a ser exactamente este valor. Un alumno hace referencia a la pregunta 1 del $C_{1}$ para justificar que la serie converge a un número menor pero muy cercano a 1 . Dos alumnos identifican y resuelven erróneamente la serie, tratando de usar conocimientos de series.

b) El proceso infinito es completo.

Catorce estudiantes afirman que el valor de la suma infinita es 1. Dos de éstos resuelven la serie correctamente resolviendo el límite de las sumas parciales. Cuatro alumnos justifican, haciendo referencia al proceso explícito de «divergencia» y «convergencia»: «a medida que se va sumando se tienen sumandos más pequeños», «los términos de la serie decrecen y tienden a $0 » ; \mathrm{y}$, cuatro estudiantes representan la situación como un cuadrado de lado 1 y lo van dividiendo.

c) No se puede determinar la suma o el valor de la suma es infinito.

Siete estudiantes afirman que el valor de la suma no se puede determinar. Dos de éstos afirman que es por la infinitud de la suma, tres por verla como una serie divergente y uno por escribir erróneamente la fórmula del n-ésimo término. Cuatro alumnos afirman que la suma es infinita. Identifican o resuelven erróneamente la serie, tratando de usar conocimientos de series, cuatro alumnos.

En la siguiente tabla se puede observar la relación o no-relación que los estudiantes establecen con los conocimientos de series o relacionando con otra pregunta del cuestionario.

\begin{tabular}{|c|c|c|c|}
\hline & $\begin{array}{c}\text { No escribe la } \\
\text { suma como } \\
\text { serie, no tiene } \\
\text { en cuenta } \\
\text { conocimientos } \\
\text { de series } \\
\text { explícitamente }\end{array}$ & $\begin{array}{c}\text { Comenta la } \\
\text { relación con } \\
\text { otra u otras } \\
\text { preguntas del } \\
\text { cuestionario }\end{array}$ & $\begin{array}{c}\text { Calcula la serie } \\
\text { demostrando la } \\
\text { convergencia } \\
\text { a través del } \\
\text { límite de } \\
\text { las sumas } \\
\text { parciales }\end{array}$ \\
\hline $\begin{array}{c}\text { Núm. de } \\
\text { alumnos }\end{array}$ & 22 & 3 & 2 \\
\hline
\end{tabular}

\section{Pregunta 4}

a) El proceso infinito no es completo, la función tiende (se acerca, se aproxima) a 2, 0 o 1.

Algunos estudiantes confunden la recta $y=2$, con la recta $y=0$ y afirman que la función tiende (se acerca, se aproxima) a 0 (un estudiante afirma que a 1). Éstos son 28 estudiantes. Veintinueve observaron correctamente la recta $y=2$ y se encuentran en esta categoría de respuesta. Dos de éstos explicitan que la función nunca será 2. Un alumno afirma que la función se acerca a 1 .

b) El proceso infinito es completo.
Dieciocho alumnos se encuentran en esta categoría y afirman que el valor de la función es 2 . Tres visualizan que tiende a transformarse en una recta. Cinco explicitan que $f(x)=2$ en el infinito, y otro, cuando se «hace extremadamente grande». La mayoría expresa que la función tiende (se acerca) a 2, además de afirmar que su valor es 2 .

c) La función es infinita

Tres estudiantes se encuentran en esta categoría. Uno de ellos considera la función como una función trigonométrica y por ello afirma que su valor tiende a infinito. No percibe el «movimiento» del rango, como proceso «divergente».

d) No se puede determinar el valor.

Dos alumnos afirman que no se puede determinar el valor de la función y afirman que $f(x)$ tiende a 0 .

A continuación se puede observar el número de alumnos que hacen referencia, o no a otros conceptos asociados, o a teorías matemáticas.

\begin{tabular}{|l|c|c|c|}
\hline & $\begin{array}{c}\text { No explicita } \\
\text { ningún concepto } \\
\text { ni se refiere } \\
\text { a teorías } \\
\text { matemáticas }\end{array}$ & $\begin{array}{c}\text { Hace } \\
\text { referencia } \\
\text { y usa } \\
\text { el símbolo } \\
\text { de límite }\end{array}$ & $\begin{array}{c}\text { Trata de justificar } \\
\text { matemáticamente } \\
\text { la situación }\end{array}$ \\
\hline $\begin{array}{c}\text { Núm. de } \\
\text { alumnos }\end{array}$ & 69 & 6 & 1 \\
\hline
\end{tabular}

\section{Pregunta 2}

No tomamos en cuenta si la respuesta fue calculada o no, sino la percepción actual o no de la situación planteada. Como se puede observar en la figura del anexo III, hubo particular dificultad para resolver este problema.

a) El proceso infinito no es completo, pero acotado.

Doce estudiantes se encuentran en esta categoría, y responden unos afirmativamente (8 estudiantes) y otros negativamente ( 5 alumnos) a la cuestión. Como se observa en la figura del anexo III, las razones han sido variadas. Se afirma (1 alumno) que esta situación no se puede experimentar realmente pero que, a partir de una serie al infinito, se obtiene un valor aproximado. Dos alumnos argumentan que es por la misma razón de la pregunta 1 que sólo se puede dar un aproximado. Algunos (cuatro) dan un valor aproximado, dos de éstos tratan de escribir la serie que representa la situación. Otros dos afirman que, hallando una serie, se puede dar ese valor aproximado.

b) El proceso es infinito y no acotado.

Tres estudiantes afirman que la pelota no termina de rebotar y dos que no se puede calcular la distancia porque es infinita.

c) El proceso infinito es completo. 
Doce alumnos respondieron afirmativamente a la pregunta y dieron una distancia. Siete de éstos escribieron la distancia dejándola indicada como una serie, por ejemplo: $\left\langle D=4 \Sigma 1 / 2^{n} »\right.$. Interpretamos que el estudiante aquí acepta la suma como un límite, como un «objeto», la cual da como resultado la distancia recorrida de la pelota.

\section{d) El proceso depende del mundo físico/finito.}

Veinticuatro estudiantes, como se pude observar en la figura del anexo III usaron argumentos finitos o relacionados con el mundo físico para contestar la pregunta a pesar del proceso infinito implícito a la pregunta. Un alumno escribe una suma finita y otro argumenta la posibilidad de calcular la distancia recorrida por la pelota, por la finitud de la distancia total. El resto considera que, teniendo datos físicos, es posible calcular la distancia, que los agentes externos paran la pelota, que en el mundo real se puede calcular, etc.

En la siguiente tabla se puede observar el número de alumnos que, en sus argumentos, hacen referencia a la teoría de series y que relacionan esta pregunta con la primera del cuestionario.

\begin{tabular}{|c|c|c|}
\hline & $\begin{array}{c}\text { Escribe una serie. Hace } \\
\text { referencia a series }\end{array}$ & $\begin{array}{c}\text { Relaciona con la } \\
\text { pregunta 1 }\end{array}$ \\
\hline $\begin{array}{c}\text { Núm. } \\
\text { de alumnos }\end{array}$ & 16 & 2 \\
\hline
\end{tabular}

\section{Algunos resultados}

Hemos podido observar, a través de las redes sistémicas, que la mayoria de los estudiantes responden afirmativamente o negativamente a las preguntas: $a$ ) dejándose llevar por la intuición o por la contextualización de la pregunta; $b$ ) con argumentos que recurren a la división infinita en mitades, posibilidad de poder dividir infinitamente, o a la existencia de infinitos puntos en una recta; $c$ ) sumando o aproximando valores; $d$ ) aceptando, sin demostración, la convergencia o divergencia de la serie $\Sigma 1 / 2^{n}$. En particular, de los 89 alumnos sólo 2 de éstos prueban, a partir del cálculo del límite de las sumas parciales, que $\Sigma 1 / 2^{n}=1$, y ninguno usa alguna prueba de convergencia. En general usan pocos argumentos matemáticos formales para responder a las cuestiones y es un menor número de alumnos los que nombran, en sus argumentos, los conceptos de límite, sucesiones o series. Sólo 5 alumnos relacionan su respuesta con alguna otra del cuestionario.

Ya se ha hecho referencia a que las cuatro preguntas presentan una situación similar, cognitivamente hablando. Los alumnos muestran que, si bien saben calcular una suma infinita (aunque no calculen la suma), reconocen si es convergente o divergente, y conocen y saben calcular un límite al infinito; algunos aceptan la situación de que en el infinito se alcanza al punto límite y otros, no. El proceso infinito implícito para algunos alumnos es completo, para otros no lo es y un menor número man- tuvo esquemas finitos o físicos a pesar de la infinitud del proceso.

En la siguiente sección, haciendo uso de las líneas de coherencia (Garbin, 2000; Garbin y Azcárate, 2002), podemos ver que la percepción, potencial, actual o finitista, de las situaciones matemáticas planteadas no se mantienen estables en las respuestas de los estudiantes, a pesar de los conocimientos de límites, sucesiones y series de los estudiantes.

\section{Líneas de coherencia}

Se elabora, con los datos que presentan las redes sistémicas, un instrumento de análisis, que se ha llamado líneas de coherencia, construido con la metodología de Garbin (2000) y Garbin y Azcárate (2002). Para elaborarlas se tiene en cuenta el concepto involucrado, las respuestas de los alumnos representadas en unas tablas resumen, derivadas de las redes sistémicas, y el contexto de las mismas, expresado en los lenguajes matemáticos distintos (geométrico, verbal, analítico, gráfico y algebraico). Además, como se afirmó en el marco teórico, se toman en cuenta también los procesos explicitados por Nuñez (1994). Se han establecido tres líneas: línea finitista (o de evasión de infinitud), línea actual y línea potencial. En el gráfico 2, a modo de ejemplo, se puede observar la línea 1; el resto, se observa en los anexos. Estas líneas representan aquellas respuestas (correctas o erróneas) que han representado una percepción del proceso infinito, como acabado, no acabado o evadido por el contexto físico o por mantener esquemas finitos ante las cuestiones. Estos gráficos están formados por tablas entrelazadas entre sí. Cada tabla tiene un enunciado que corresponde a las respuestas dadas por los alumnos y debajo del enunciado aparecen los números correspondientes a los alumnos que han dado dicha respuesta. Por tanto, se puede observar la frecuencia de alumnos por respuesta y, si se sigue el orden dado por los enlaces, se pueden identificar aquellos estudiantes que mantienen o no sus respuestas con una concepción actual, potencial o finitista de las situaciones planteadas.

En Garbin (2003) se establecieron 4 grupos de respuestas: finitista (1), actual (2), potencial (3) y grupo mixto. El criterio para la conformación de los grupos es: si un alumno tiene tres o más respuestas en una línea determinada, se le sitúa en el grupo de la línea correspondiente. Es decir, si un alumno tiene tres o más respuestas en la línea 3, se le sitúa en el grupo 3, que corresponde al grupo potencial. En la siguiente tabla podemos observar el número y el porcentaje de alumnos por grupo:

\begin{tabular}{|c|c|c|c|c|}
\hline Grupo & $\mathbf{1}$ & $\mathbf{2}$ & $\mathbf{3}$ & Mixto \\
\hline $\begin{array}{c}\text { Núm. de } \\
\text { alumnos }\end{array}$ & 3 & 12 & 32 & 42 \\
\hline$\%$ & 3,37 & 13,48 & 35,95 & 47,19 \\
\hline
\end{tabular}


Gráfico 2

Línea 1. Línea finitista.

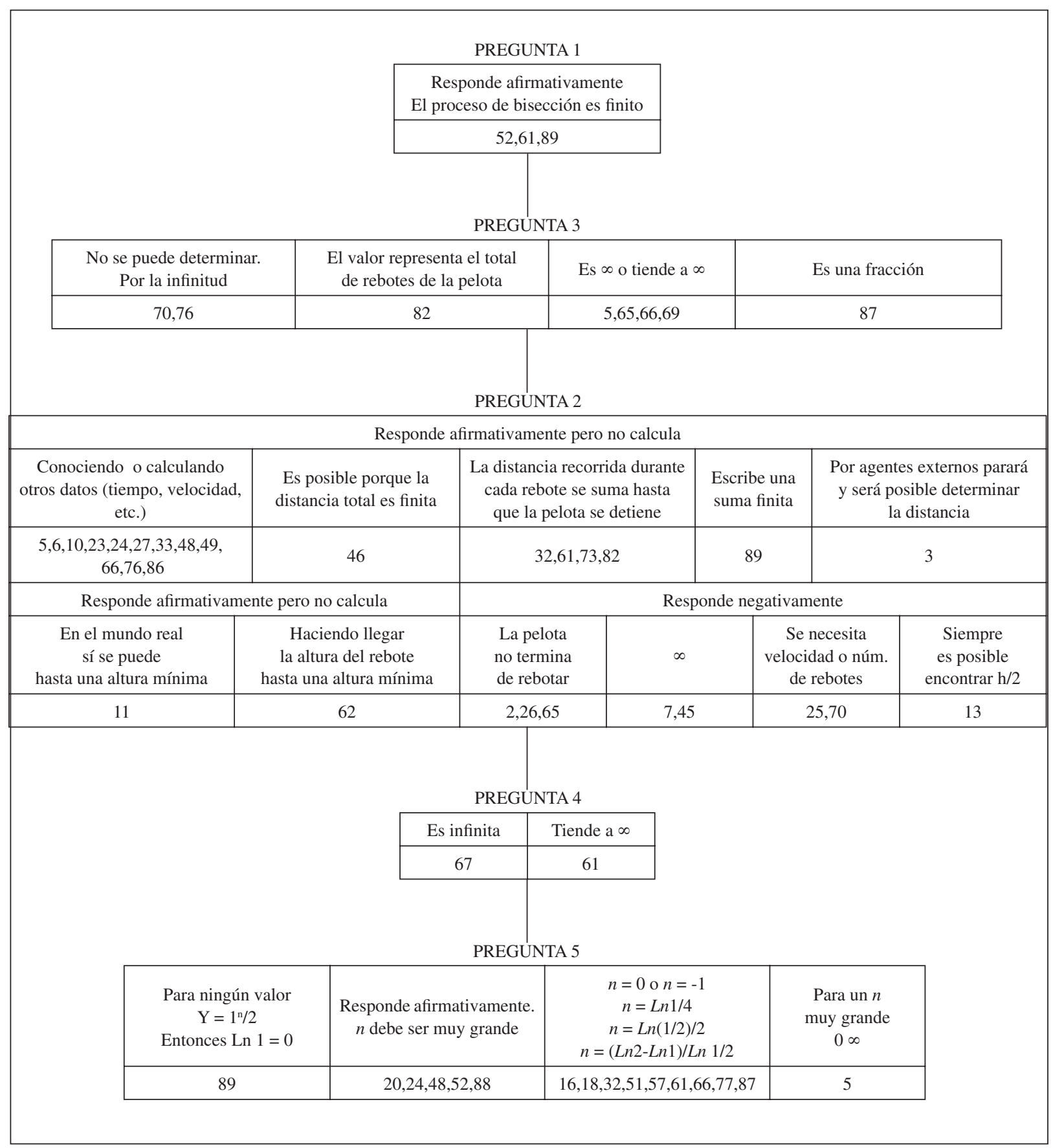

De cada grupo se pueden establecer unas subcategorías que permiten observar el número de respuestas «actualistas», «finitistas (o de evasión)» o «potenciales». Es decir, un alumno pudo tener cinco respuestas en la línea actual (5A) o tres en la línea actual, una en la línea finitista (o de evasión) y dos en la línea potencial. Esta situación se representa simbólicamente como $3 \mathrm{~A}+1 \mathrm{~F}+2 \mathrm{P}$. En la siguiente tabla se observan las subcategorías y entre paréntesis los números que identifican a los alumnos que pertenecen a cada subcategoría. Ejemplo: el alumno número 61 está ubicado en el grupo 1 , cuatro de sus respuestas fueron «finitistas» (4F) y una «potencial» (1P). Si se desea saber a qué preguntas corresponden dichas respuestas, se observa la línea de coherencia respectiva. 


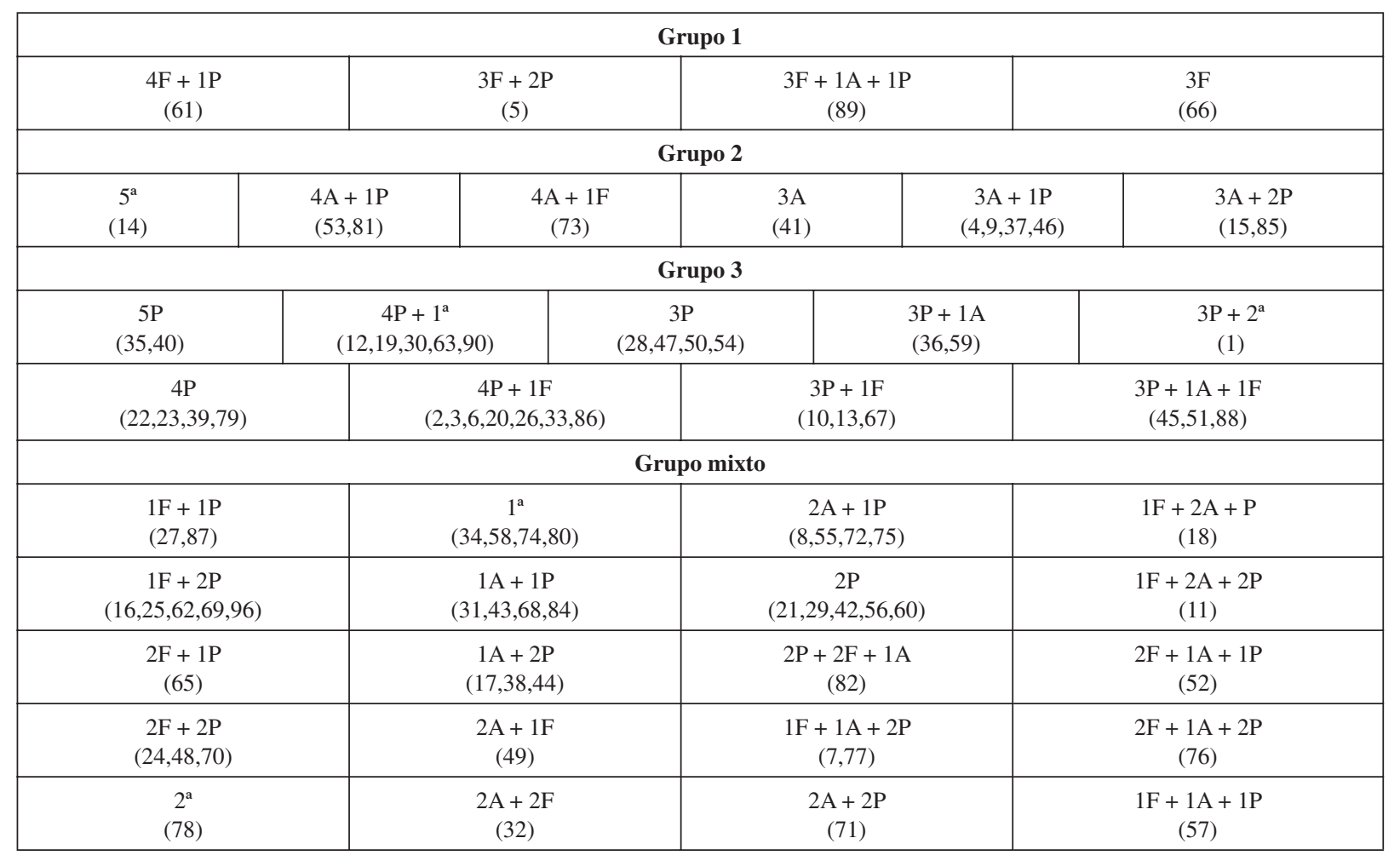

Se nota, entre otras cosas, que hay un solo estudiante que, en todas sus respuestas, acepta que el proceso infinito es acabado (grupo 2) y 2 alumnos, en el grupo 3, que mantienen una concepción potencial del infinito. Un alto número de estudiantes se encuentra en el grupo mixto.

Los distintos contextos de las preguntas y conceptos matemáticos asociados a las mismas determinan percepciones distintas en la completes o no del proceso, esto a pesar de los conocimientos del cálculo diferencial e integral de los estudiantes encuestados. Un hecho interesante es que en el grupo 1, que son estudiantes que mantienen principalmente esquemas finitos, no aparece la posibilidad de la completes del proceso en ninguna de las preguntas. En caso contrario, en el grupo donde sí aparece y se acepta alcanzar al punto límite, no hay casi presencia de respuestas finitistas (o de evasión de finitud), sólo un estudiante presenta una respuesta de este tipo.

Se hace evidente la naturaleza conflictiva de las intuiciones del infinito, la influencia de los lenguajes y contextos en las percepciones de los estudiantes, y la persistencia de la imagen informal aunque ciertas ideas formales hayan sido presentadas a los estudiantes.

\section{Conexiones y relaciones que establecen los estu-} diantes entre las preguntas 1,3 y 4

Distintos contextos requieren un foco de atención sobre diversos aspectos y es importante filtrar aquéllos que no dejan hacer matemáticas (Pitta y Gray, 1997; citado en
Gray et al., 1999). En el cuestionario $\mathrm{C}_{2}$, se pide a los alumnos que definan, si existen, una o varias relaciones (conexiones, similitudes) entre los tres problemas (planteamiento y resolución), preguntas 1,3 y 4 de $C_{1}$. Se analizan y se clasifican las respuestas en grupos no excluyentes.

Los estudiantes expresan las relaciones de similitud entre los tres problemas y focalizan su atención en distinto aspectos (Garbin, 2003): a) El planteamiento del problema: la respuesta del alumno está en relación con lo que plantean los problemas o con lo que se pide hacer: A (33) ${ }^{1}$ : «Supongo que el planteamiento de los tres casos es probar el concepto de tendencia. Examinar si para el estudiante es teóricamente posible que si una sucesión, una suma, o fracción tiende a un número determinado, llegará eventualmente a tomar el valor de dicho número. Para mí no es así»; A (59): «Los tres problemas son distintas maneras de plantear la misma situación, una suma infinita de términos que decrecen. La primera es la forma geométrica, luego una forma analítica (sólo álgebra) y después en forma gráfica». b) El proceso de convergencia y divergencia implícito en cada pregunta (aceptando o no la situación límite): A (16): «las funciones van haciendo que sea un valor más y más pequeño, además de que su planteamiento es un valor que a medida que crece, genera un resultado que tiende a otro valor más pequeño». c) El concepto implícito, la noción de infinito o el reconocimiento que se trata del mismo concepto o tema en todas las preguntas: $\mathrm{A}(38)$ : «los tres se relacionan con el infinito»; $\mathrm{A}(82)$ : «En los tres problemas se plan- 
tean que, para llegar a un resultado, hay que tener en cuenta el $\infty$ matemático [...]»; A(3): «La pregunta es la misma, el concepto que se quiere «evaluar» es el mismo para las tres preguntas». d) Los conceptos asociados de límites, sucesiones o series: A(9): «Tienen como fundamento las definiciones de límites, sucesiones y series». e) Resultados: puede ser el tipo de resultado o el procedimiento o modo de resolución del problema: $\mathrm{A}(22)$ : «la resolución de los tres en forma exacta es imposible»; $\mathrm{A}(8)$ : «se resuelven similarmente». $f$ ) Encuentran similitud en algunas o detallan semejanzas y diferencias entre los problemas: A(12): «Sólo hay similitud entre el problema 1 y 2 a manera de fracciones»; A(75): «Entre el primer problema y el segundo hay una similitud, pues hay un término que se va dividiendo a la mitad y el siguiente término es cada vez más pequeño. Sin embargo, en el primer problema tenemos una sucesión y los puntos de la recta se van acercando a un límite que sería el extremo $B$, mientras que en el segundo problema tenemos una serie armónica cuyos términos se van sumando sin converger a un valor (su límite tiende $a \infty$ o no existe). En el tercer ejemplo tenemos una función que tiene límite igual a $2 y$, al tener límite, podemos decir que tiene una similitud con la sucesión del problema $1 »$.

En la siguiente tabla se puede ver el número de alumnos (en la etiqueta de cada grupo) y los alumnos por cada grupo clasificado. La negrita indica que estos alumnos no estan sólo en ese grupo.

\begin{tabular}{|c|c|c|c|c|c|c|c|c|c|c|}
\hline \multicolumn{2}{|c|}{$\begin{array}{l}\text { Planteamiento } \\
\text { del problema }\end{array}$} & \multicolumn{9}{|c|}{ Conceptos asociados } \\
\hline $\begin{array}{l}\text { Planteamiento } \\
\text { (Finalidad) } \\
\text { (7) }\end{array}$ & $\begin{array}{l}\text { Lenguajes } \\
\text { matemáticos } \\
\text { diferentes } \\
\text { (4) }\end{array}$ & \multicolumn{2}{|c|}{$\begin{array}{l}\text { Límite, } \\
\text { sucesión } \\
\text { y serie } \\
\text { (1) }\end{array}$} & $\begin{array}{l}\text { Límite } \\
\text { (8) }\end{array}$ & $\begin{array}{l}\text { Serie } \\
\text { (8) }\end{array}$ & $\begin{array}{l}\text { Sucesión } \\
\text { (4) }\end{array}$ & $\begin{array}{c}\text { Serie y } \\
\text { sucesión } \\
\text { (7) }\end{array}$ & $\begin{array}{l}\text { Suma } \\
\infty \\
\text { (3) }\end{array}$ & $\begin{array}{l}\text { Función } \\
\text { expo- } \\
\text { nencial } \\
\text { (1) }\end{array}$ & $\begin{array}{l}\text { Infinitesi- } \\
\text { males } \\
\text { (1) }\end{array}$ \\
\hline $\begin{array}{l}(\mathbf{2})(\mathbf{2 1})(33) \\
(\mathbf{6 0})(81)(83) \\
\quad(85)\end{array}$ & $\begin{array}{l}(\mathbf{9})(\mathbf{1 9}) \\
(59)(69)\end{array}$ & & 9) & $\begin{array}{c}(1)(2) \\
(24) \\
(25) \\
(35) \\
(50) \\
(62) \\
(\mathbf{8 8})\end{array}$ & $\begin{array}{l}(14) \\
(17) \\
(29) \\
(39) \\
(40) \\
(46) \\
(80) \\
(90) \\
\end{array}$ & $\begin{array}{l}(34) \\
(36) \\
(51) \\
(77)\end{array}$ & $\begin{array}{l}(\mathbf{1 3}) \\
(\mathbf{3 0}) \\
(43) \\
(44) \\
(53) \\
(55) \\
(61)\end{array}$ & $\begin{array}{l}(5) \\
(8) \\
(47)\end{array}$ & (22) & (17) \\
\hline \multicolumn{5}{|c|}{$\begin{array}{c}\text { Proceso implícito de convergencia } \\
\text { y divergencia } \\
\end{array}$} & \multicolumn{6}{|c|}{ Concepto implícito } \\
\hline $\begin{array}{l}\text { Acepta situación límite } \\
\text { (3) }\end{array}$ & \multicolumn{2}{|c|}{$\begin{array}{c}\text { No acepta situación } \\
\text { límite (1) }\end{array}$} & \multirow{2}{*}{\multicolumn{2}{|c|}{$\begin{array}{c}\text { No alude a la } \\
\text { situación límite } \\
(9) \\
(\mathbf{1 3})(16)(\mathbf{1 9}) \\
(45)(52)(\mathbf{6 7}) \\
(72)(\mathbf{7 6})(\mathbf{8 8})\end{array}$}} & & \multicolumn{2}{|c|}{$\begin{array}{l}\text { Noción de } \infty \\
\text { (3) }\end{array}$} & \multicolumn{3}{|c|}{$\begin{array}{c}\text { El mismo concepto o teoría } \\
\text { matemática (2) }\end{array}$} \\
\hline$(11)(15)(32)$ & \multicolumn{2}{|l|}{ (70) } & & & & \multicolumn{2}{|c|}{$(\mathbf{2 1})(38)(82)$} & \multicolumn{3}{|c|}{$(3)(71)$} \\
\hline \multicolumn{5}{|c|}{ Resultados } & & \multirow{2}{*}{\multicolumn{5}{|c|}{$\begin{array}{l}\text { Similitud en algunas semejanzas } \\
\text { o diferencias en los lenguajes } \\
\text { (13) }\end{array}$}} \\
\hline $\begin{array}{c}\text { Tipo de resultac } \\
\text { (11) }\end{array}$ & & $\begin{array}{r}\text { Procec } \\
\text { d }\end{array}$ & $\begin{array}{l}\text { miento } \\
\text { resoluc } \\
(16)\end{array}$ & $\begin{array}{l}\text { modo } \\
\text { n }\end{array}$ & & & & & & \\
\hline \multicolumn{2}{|c|}{$\begin{array}{c}(10)(\mathbf{1 4})(\mathbf{1 7})(\mathbf{2 2})(23) \\
(\mathbf{3 9})(63)(66)(\mathbf{7 1})(\mathbf{7 6}) \\
(79)\end{array}$} & \multicolumn{3}{|c|}{$\begin{array}{c}(7)(\mathbf{8})(26)(27)(\mathbf{2 9}) \\
(37)(41)(47)(54) \\
(56)(58)(63)(\mathbf{6 7}) \\
(\mathbf{7 1})(78)(87)\end{array}$} & & \multicolumn{5}{|c|}{$(4)(6)(12)(\mathbf{1 4})(20)(\mathbf{3 0})(31)(48)(49)(\mathbf{6 0})(73)(75)$} \\
\hline
\end{tabular}


El interés de este estudio también es establecer una posible relación, si existe, entre los focos de atención que los estudiantes explicitaron al hablar de las posibles relaciones y conexiones entre los tres problemas, y la percepción del infinito (potencial-actual) que mostraron en sus respuestas.

A partir de la última tabla, se construye una nueva teniendo en cuenta a qué grupo pertenece cada alumno. De esta forma se puede observar si hay alguna relación entre aquéllos que han mostrado una tendencia mayoritaria de respuestas aceptando la completes o no del proceso involucrado en las cuestiones planteadas y las relaciones o conexiones que establecieron entre las tres preguntas de $\mathrm{C}_{2}$. La abreviatura $G r$. quiere indicar grupo (ejemplo: $G r . l^{2}(2)=$ hay dos alumnos pertenecientes al grupo 1), y Mix. quiere indicar grupo mixto (ejemplo: mixto (4) = hay 4 alumnos del grupo mixto).

Analizando la tabla y los datos a los que ella se refiere, se observa que no hay una conducta mayoritaria o relación directa que se pueda establecer entre aquellos alumnos que han mostrado respuestas finitistas, actualistas o potencialistas, y las relaciones de similitud que han expresado. Se puede observar que hay muy poca presencia de alumnos que se encuentren en el grupo actual, en las categorías de conceptos asociados, «proceso implícito de convergencia y divergencia» y del concepto implícito (4 de 50 estudiantes). Llama la atención que en la categoría «Proceso implícito de convergencia y divergencia», tres alumnos explicitan que aceptan la situación límite; sin embargo, uno sólo de éstos se encuentra en el grupo 2.

En Garbin (2000) se caracteriza la «tarea de conexión», «que consistiría en identificar y establecer relaciones entre los problemas, en cuanto a lenguaje matemático y registro de representación semiótica se refiere, y reconocer los contextos (conceptual y global) de los problemas, de manera que permita una influencia mutua dando lugar a respuestas asociadas coherentes a los problemas». La inducción de esta tarea en algunos alumnos entrevistados, deja en evidencia que ha sido «fundamental»: $a$ ) reconocer en todas las preguntas el proceso de división infinita, con los dos tipos de iteraciones, la divergente y convergente; b) establecer la relación y conexión entre las preguntas a través de la sucesión numérica. Como se puede observar en las tablas anteriores, los alumnos focalizaron su atención al describir las relaciones, conexiones o similitudes en los problemas del $\mathrm{C}_{2}$, en diferentes partes estructurales de ellos, el planteamiento, la resolución, los resultados, los conceptos asociados, etc. Sólo 13 alumnos de los 89 hacen referencia al proceso implícito de convergencia y divergencia presente en todas las preguntas. Nuevamente queda en evidencia la persistencia de la imagen informal aunque hayan sido introducidas ideas formales en la enseñanza, la cual es sensible al contexto y lenguajes matemáticos involucrados. Es probable que, en el esquema conceptual de los estudiantes, se mantengan en tensión constante el infinito «perceptual» y las imágenes formales asociadas a los conceptos formales del cálculo diferencial e integral.

\begin{tabular}{|c|c|c|c|c|c|c|c|c|c|c|}
\hline \multicolumn{2}{|c|}{$\begin{array}{l}\text { Planteamiento } \\
\text { del problema }\end{array}$} & \multicolumn{9}{|c|}{ Conceptos asociados } \\
\hline $\begin{array}{l}\text { Plantea- } \\
\text { miento } \\
\text { (Finalidad) }\end{array}$ & $\begin{array}{l}\text { Lenguajes } \\
\text { matemáticos } \\
\text { diferentes }\end{array}$ & \multicolumn{2}{|c|}{\begin{tabular}{|} 
Límite, \\
sucesión y \\
serie
\end{tabular}} & Límite & Serie & Sucesión & $\begin{array}{c}\text { Serie } \\
\text { y sucesión }\end{array}$ & $\begin{array}{l}\text { Suma } \\
\infty\end{array}$ & $\begin{array}{l}\text { Función } \\
\text { expo- } \\
\text { nencial }\end{array}$ & $\begin{array}{l}\text { Infinitesi- } \\
\text { males }\end{array}$ \\
\hline $\begin{array}{c}\text { Grupo } 2(2) \\
\text { Grupo } 3(2) \\
\text { Mixto (2) }\end{array}$ & $\begin{array}{c}\text { Grupo } 2(1) \\
\text { Grupo } 3(2) \\
\text { Mixto (1) }\end{array}$ & \multicolumn{2}{|c|}{ Gr. $2(1)$} & $\begin{array}{l}\text { Gr. } 3(5) \\
\text { Mixto (3) }\end{array}$ & $\begin{array}{l}\text { Gr. } 2(2) \\
\text { Gr. } 3 \text { (3) } \\
\text { Mix. (3) }\end{array}$ & $\begin{array}{l}\text { Gr. } 3(2) \\
\text { Mix. (2) }\end{array}$ & $\begin{array}{l}\text { Gr. } 1(1) \\
\text { Gr. } 2(1) \\
\text { Gr. } 3(2) \\
\text { Mix. (3) }\end{array}$ & $\begin{array}{l}\text { Gr. } 1 \text { (1) } \\
\text { Gr. } 3(1) \\
\text { Mix. (1) }\end{array}$ & Gr. $3(1)$ & Mixto (1) \\
\hline \multicolumn{5}{|c|}{$\begin{array}{l}\text { Proceso implícito de convergencia } \\
\text { y divergencia }\end{array}$} & \multicolumn{6}{|c|}{ Concepto implícito } \\
\hline $\begin{array}{l}\text { Acepta situación } \\
\text { límite }\end{array}$ & \multicolumn{2}{|c|}{$\begin{array}{l}\text { No acepta } \\
\text { situación límite }\end{array}$} & & $\begin{array}{l}\text { To alude a } \\
\text { ación límite }\end{array}$ & \multicolumn{3}{|c|}{ Noción de $\infty$} & \multicolumn{3}{|c|}{$\begin{array}{l}\text { El mismo concepto o teoría } \\
\text { matemática }\end{array}$} \\
\hline $\begin{array}{l}\text { Grupo } 2 \text { (1) } \\
\text { Mixto (2) }\end{array}$ & \multicolumn{2}{|c|}{ Mixto (1) } & & $\begin{array}{l}\text { upo } 3 \text { (5) } \\
\text { ixto }(4)\end{array}$ & \multicolumn{3}{|c|}{ Mixto (3) } & \multicolumn{3}{|c|}{$\begin{array}{l}\text { Grupo } 3 \text { (1) } \\
\text { Mixto (1) }\end{array}$} \\
\hline \multicolumn{5}{|c|}{ Resultados } & \multirow{2}{*}{\multicolumn{6}{|c|}{$\begin{array}{l}\text { Similitud en algunas semejanzas } \\
\text { o diferencias en los lenguajes }\end{array}$}} \\
\hline \multicolumn{2}{|c|}{ Tipo de resultado } & \multicolumn{3}{|c|}{$\begin{array}{l}\text { Procedimiento o modo } \\
\text { de resolución }\end{array}$} & & & & & & \\
\hline \multicolumn{2}{|c|}{$\begin{array}{l}\text { Grupo } 1 \text { (1) } \\
\text { Grupo } 2(1) \\
\text { Grupo } 3 \text { (6) } \\
\text { Mixto (3) }\end{array}$} & \multicolumn{3}{|c|}{$\begin{array}{l}\text { Grupo } 2(2) \\
\text { Grupo } 3(5) \\
\text { Mixto (9) }\end{array}$} & \multicolumn{6}{|c|}{$\begin{array}{l}\text { Grupo } 2(3) \\
\text { Grupo } 3(4) \\
\text { Mixto (5) }\end{array}$} \\
\hline
\end{tabular}




\section{CONCLUSIONES E IMPLICACIONES DOCEN- TES}

A partir de las redes sistémicas y de su análisis, se pudieron interpretar los esquemas conceptuales de los estudiantes en relación con la situación matemática que presentan las preguntas del $\mathrm{C}_{1}$, las cuales fueron descritas en la primera parte del artículo. Los estudiantes perciben y muestran, en sus respuestas, una concepción del infinito que no se mantiene a lo largo de las preguntas. En algunas de éstas, el proceso infinito no es percibido como acabado y en otras, sin embargo, sí es aceptada la completez del proceso. A pesar de la edad y de los conocimientos previos, saber calcular una suma infinita (aunque no se calcule la suma), reconocer si es divergente o convergente y conocer y saber calcular un límite al infinito, muestra en algunos que aceptan la situación de que el infinito se alcanza en el punto límite y en otros no. Siguen apareciendo respuestas finitistas a las cuestiones y, en algunas preguntas, la contextualización físico/finita influye en evadir la infinitud.

Hemos visto y subrayado que la mayoría de los estudiantes, indistintivamente a si aceptan la situación límite o no de las preguntas, argumentan sus respuestas: $a$ ) dejándose llevar por la intuición o por la contextualización de las pregunta; $b$ ) con argumentos que recurren a la división infinita en mitades, posibilidad de poder dividir infinitamente o a la existencia de infinitos puntos en una recta; $c$ ) sumando o aproximando valores; $d$ ) aceptando sin demostración la convergencia o divergencia de la serie $\Sigma 1 / 2^{n}$. En particular, de los 89 alumnos, sólo 2 de éstos prueban, a partir del cálculo del límite de las sumas parciales, que $\Sigma 1 / 2^{n}=1$, y ninguno usa alguna prueba de convergencia. En general usan pocos argumentos matemáticos formales para responder a las cuestiones y es un número pequeño de alumnos los que nombran en sus argumentos los conceptos de límite, sucesiones o series. Sólo 5 alumnos relacionan su respuesta con alguna otra del cuestionario.

Las líneas de coherencia permiten observar el comportamiento de las respuestas de los alumnos y establecer unos grupos de respuestas con sus respectivas subcategorías. Hemos visto que un solo estudiante en todas sus respuestas acepta que el proceso infinito implicado en las cuestiones es acabado, completo, y dos alumnos mantienen en todas las respuestas una concepción potencial del infinito. La mayoría de los estudiantes dieron respuestas «mixtas» (en el sentido que hemos descrito en el artículo). La «imagen formal» de los conceptos asociados de límites, series, sucesiones, etc. aparentemente también entró en conflicto con la «imagen informal» reflejada en cada situación, particularmente por el elemento intuitivo y contraintuitivo de las preguntas. La «imagen informal» persiste en los esquemas conceptuales de los alumnos aunque hayan sido introducidas ideas formales durante la enseñanza.

A partir del segundo cuestionario se pudo establecer una clasificación de seis grupos (no excluyentes) de respuestas, que muestran, en parte, qué tipo de conexiones reconocen y establecen los estudiantes entre tres de los problemas planteados en el estudio. Esta clasificación evidencia que reconocer o establecer conexiones «fundamentales» entre un problema, que presenta una «misma» situación cognitiva, pero representado en diferente forma, no es una habilidad espontánea y que debe ser adquirida. El docente debe ser consciente de que el estudiante, ante un «mismo problema» pero representado en diferentes lenguajes, puede o no reconocer este hecho, así como puede reconocer sólo algunos de los aspectos estructurales de semejanza de los problemas, como los clasificados. Las similitudes y diferencias, en cuanto a planteamiento, concepto implícito, conceptos asociadas, procedimiento de resolución y tipos de resultados, deben ser inducidos durante la práctica docente, así como la habilidad de reconocer aquellas conexiones «fundamentales» que generen respuestas asociadas coherentes.

Otro interés del estudio era establecer una posible relación, entre los focos de atención y conexiones y la percepción del infinito que los estudiantes evidenciaron. A partir de las clasificaciones realizadas y tablas diseñadas, se observa que no hay conducta mayoritaria o relación directa que se pueda establecer entre aquellos alumnos que han mostrado respuestas del tipo «finitistas», «actualistas» o «potencialistas», y las relaciones de similitud entre las preguntas que han expresado.

Todos estos resultados inducen a pensar que el conocimiento previo del cálculo diferencial o integral es de ayuda, pero no de una manera significativa o determinante, para establecer y reconocer las conexiones «oportunas» $y$ «fundamentales» entre los problemas planteados, así como de potenciar la noción del infinito actual. Además, como afirma Tall, se hace necesario reconstruir conceptos y conexiones en el esquema conceptual para buscar la coherencia deseada de la imagen formal.

\section{NOTA}

${ }^{1}$ A(33) indica: Alumno número 33. Se mantiene esta notación para hacer referencia a los estudiantes. 


\section{REFERENCIAS BIBLIOGRÁFICAS}

BLISS, J., MONK, M. y OGBORN, J. (1983). Qualitative Data Analysis for Educational Research. Londres: Coom Helm.

D'AMORE, B. (1996). L'infinito: storia di conflitti, di sorprese, di dubbi. La Matematica e la sua Didattica, 3, pp. 322-335.

D'AMORE, B. (1997). L'infinito in didattica della matematica. La Matematica e la sua Didattica, 3, pp. 289-305.

FISHBEIN, E. (1978). Intuition and mathematical education. Osnabrücker Schriften zür Mathematik, 1, pp. 148-176.

FISHBEIN, E. (1982). Intuition and proof. For the Learning of Mathematics, (3)2, pp. 9-19.

FISHBEIN, E. (2001). Tacit models and infinity. Educational Studies in Mathematics, 2-3(48), pp. 309-329.

FISHBEIN, E., TIROSH, D. y HESS, P. (1979). The intuition of infinity. Educational Studies in Mathematics, 10, pp. 2-40.

FISHBEIN, E., TIROSH, D. y MELAMED, U. (1981). It is possible tu measure the intuitive acceptance of mathematical statament? Educational Studies in Mathematics, 12, pp. 491-512.

GARBIN, S. (2000). «Infinito actual: inconsistencias e incoherencias de estudiantes de 16-17 años». Tesis doctoral. Universitat Autònoma de Barcelona.

GARBIN, S. y AZCÁRATE, C. (2000). Esquemas conceptuales e incoherencias con relación al concepto de infinito actual. Educación Matemática, 12(3), pp. 5-17.

GARBIN, S. y AZCÁRATE, C. (2002). Infinito actual e inconsistencias: Acerca de las incoherencias en los esquemas conceptuales de alumnos de 16-17 años. Enseñanza de las Ciencias, 20(1), pp. 87-113.

GARBIN, S. (2003). Incoherencias y conexiones: el caso del infinito actual con estudiantes universitarios. Primera fase del estudio. Acta Latinoamericana de Matemática Educativa (Relme 16), 16.
GRAY, E. et al. (1999). Knowledge Construction and Diverging Thinking in Elementary \& Advanced Mathematics, en Tirosh (ed.). Forms of Mathematical Knowledge, pp. 11133. Kluwer Academic Publishers.

KIDRON, I. (2002). Concept Definition, Concept Image and the notion of infinite sum in old and new environments. PME 26, 3, pp. 209-216.

MORENO, L.E. y WALDEGG, G. (1991). The conceptual evolution of actual mathematical infinity. Educational Studies in Mathematics, 22 (3), pp. 211-231.

NUÑEZ, E. (1994). Subdivision and small infinities: Zeno, paradoxes and cognition. Actas del PME 18, 3, pp. 368-375.

RUCKER, R. (1995). Infinity and the Mind. Princeton University Press.

TALL, D. (2001). Natural and Formal Infinities. Educational Studies in Mathematics, 48 (2,3), pp. 199-238.

TALL, D. y VINNER, S. (1981). Concept image and concept definition in mathematics with particular references to limits and continuity. Educational Studies in Mathematics, 12 (2), pp. 151-169.

TALL, D. y TIROSH, D. (2001). Infinity - The never- ending struggle. Educational Studies in Mathematics, 2-3 (48), pp. 129-136.

TIROSH, D. (1991). The role of students' intuitions of infinity in teaching the cantorian theory en Tall, D. (ed.). Advanced Mathematical Thinking, pp. 199-214. Dordrecht, Boston, Londres: Kluwer Academic Publisher.

TSAMIR, P. y TIROSH, D. (1995). Metacognizione e coerenza: il caso dell'infinito. La matemática e la sua didattica, 2 , pp. $122-131$.

TSAMIR, P. y TIROSH, D. (1999). Consistency and Representations: The Case of Actual Infinity. Journal for Research in Mathematics Education, 30 (2), pp. 213-219. 


\section{ANEXO I}

\section{Primer cuestionario $\left(\mathbf{C}_{1}\right)$}

1) Observa la siguiente figura.

Nos muestra un esquema en el que se bisecciona cada vez el segmento de la derecha; es decir, los puntos M, N, O, P son los puntos medios de los segmentos $\mathrm{AB}, \mathrm{MB}, \mathrm{NB}$ y $\mathrm{OB}$ respectivamente.

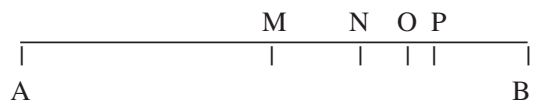

Si se siguen haciendo más y más bisecciones, ¿crees que es posible llegar a una situación en la que un punto de la bisección coincide con el punto $\mathrm{B}$ ?

Explica tu respuesta de forma detallada.

2) Se deja caer una pelota desde 2 metros de altura sobre una superficie horizontal. Cada vez que la pelota llega al suelo, tras caer desde una altura $h$, rebota hasta una altura $h / 2$.

a) ¿Podrías calcular la distancia total recorrida por la pelota? Explica tu respuesta de forma detallada.

b) ¿Podrías decir cuántos rebotes hará la pelota? Explica tu respuesta de forma detallada.

3) Considera la siguiente suma $1 / 2+1 / 4+1 / 8+1 / 16+\ldots+\ldots$

¿Cuál crees que es el valor de la suma? Explica tu respuesta de forma detallada.

4) La siguiente figura representa la gráfica de una función.

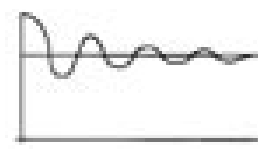

Describe lo que pasa con la función para valores muy grandes de $x$.

¿Podrías determinar el valor de la función cuando $x$ se hace extremadamente grande? Explica tu respuesta con detalle.

5) Considera la siguiente ecuación: $y=1+1 / 2+1 / 2^{2}+1 / 2^{3}+1 / 2^{4}+\ldots+1 / 2^{n}+\ldots$

¿Podrías decir para qué valor de $n$ resulta $y=2$ ?

Explica tu respuesta en forma detallada.

\section{Segundo cuestionario $\left(\mathbf{C}_{2}\right)$}

La primera página del cuestionario presenta nuevamente las preguntas 1,3 y 4 del $\mathrm{C}_{1}$. Aparece la siguiente indicación: Lee los tres problemas y contesta a las preguntas que aparecen en las páginas siguientes:

1) Si existen, define una o varias relaciones (conexiones, similitudes) entre los tres problemas (planteamiento y resolución) presentados en la página anterior. Escribe detalladamente. 


\section{ANEXO II}

\section{Pregunta 1}

El punto de bisección coincidirá con el punto extremo B? (CB)

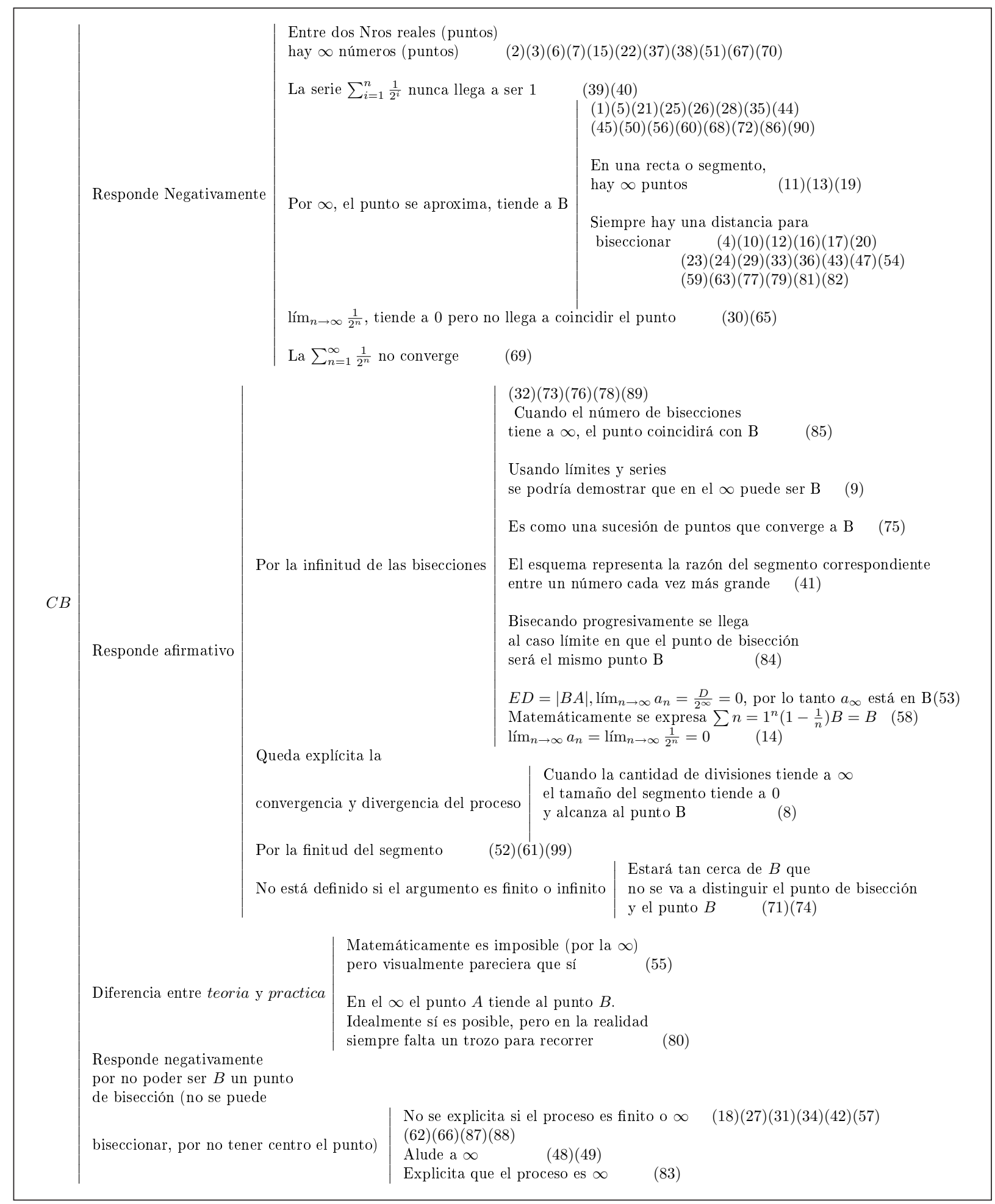




\section{ANEXO III}

Pregunta 2

Podrías calular la distancia total recorrida? (DR)

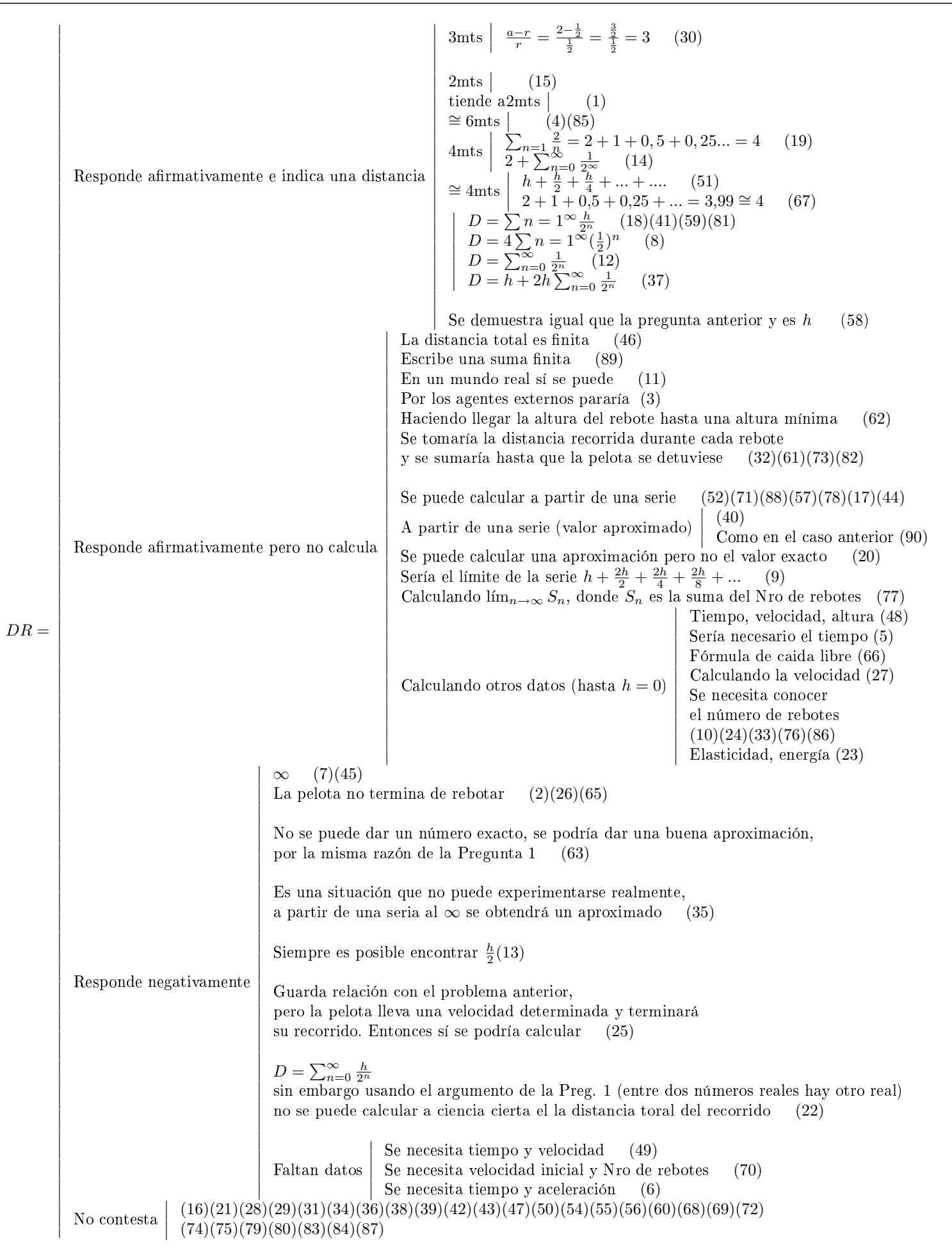




\section{ANEXO IV}

Pregunta 3

¿Cuál es el valor de la suma? (VS)

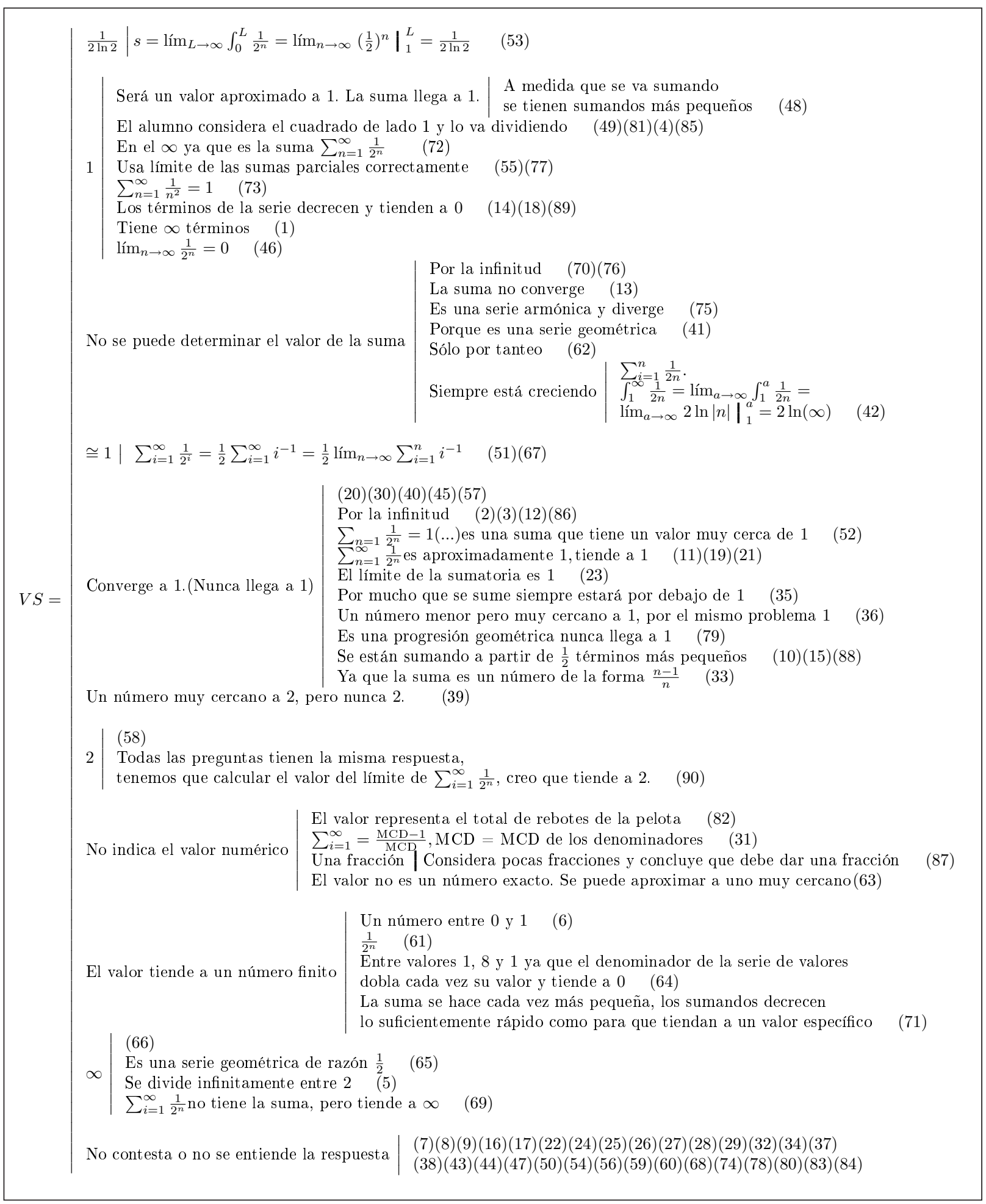




\section{ANEXO V}

Pregunta 5

¿Para qué valor de $\mathrm{n}$ resulta $\mathrm{y}=2 ?(\mathrm{VN})$

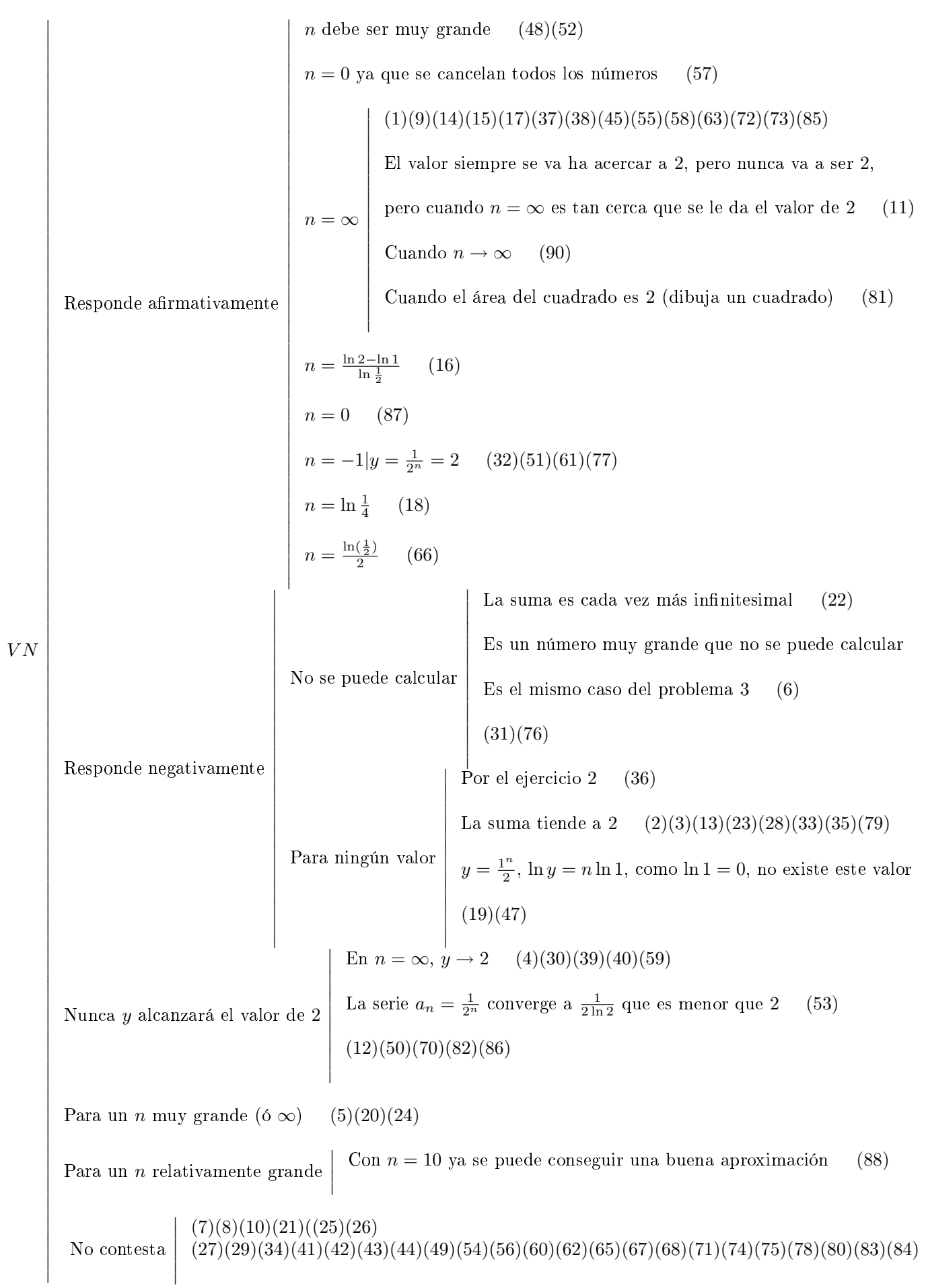




\section{ANEXO VI}

Línea 2. Línea actual

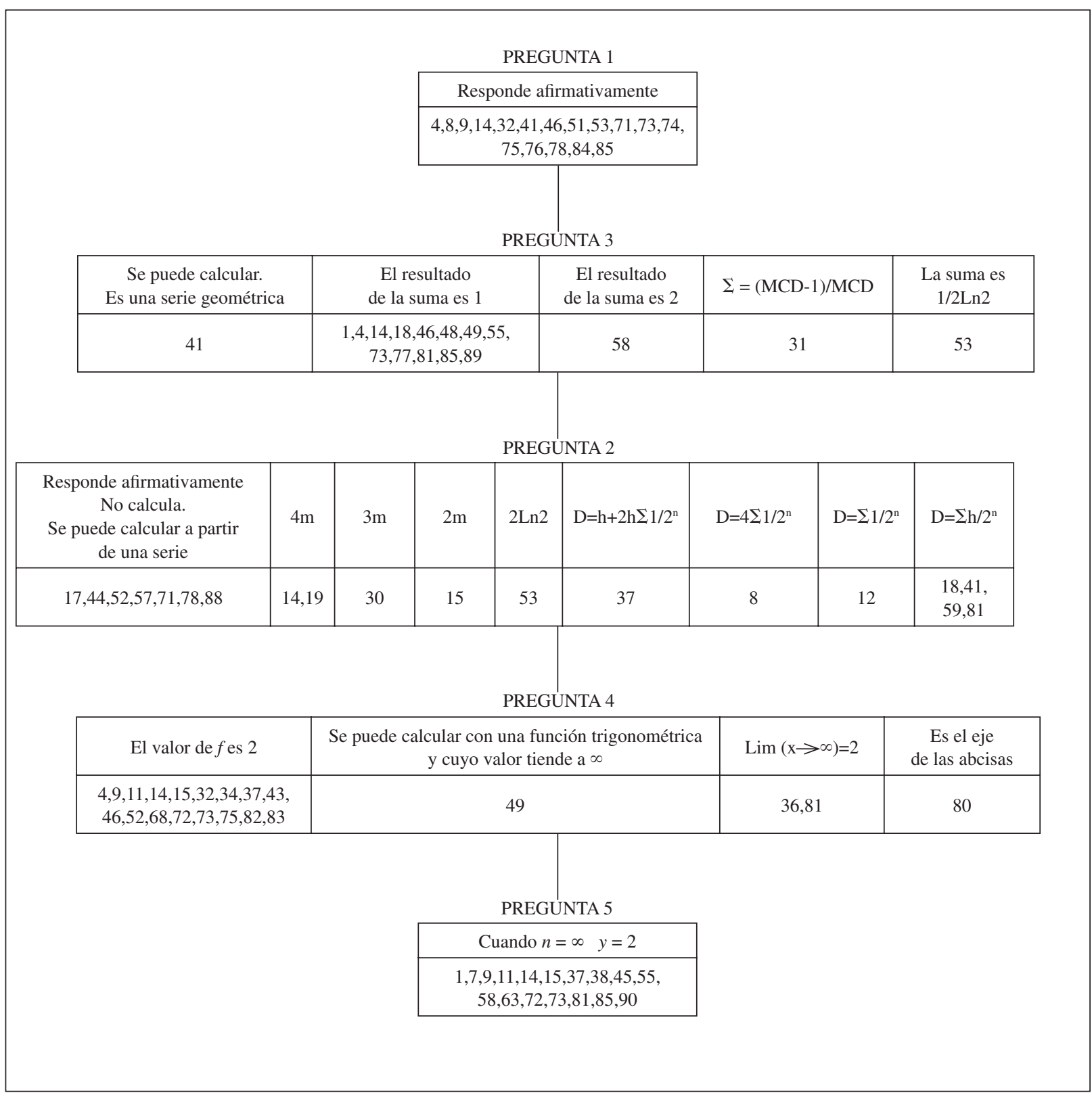




\section{ANEXO VII}

\section{Línea 3. Línea potencial}

\section{PREGUNTA 1}

\begin{tabular}{|c|c|c|}
\hline Responde afirmativamente & \multicolumn{2}{|c|}{ Responde negativamente } \\
\hline $\begin{array}{c}\text { Se redondea la cantidad } \\
\text { por la finitud de la recta }\end{array}$ & $\begin{array}{c}\text { Por la infinitud del proceso, } \\
\text { tiende o se aproxima a B }\end{array}$ & Por la mitad de la distancia \\
\hline & $1,3,4,5,6,7,10,11,12,13,15,16,17$, & \\
\hline 67 & $19,20,22,23,24,25,26,28,29,30,33$, & \\
& $35,36,37,38,39,40,43,44,45,47,50$, & \\
& $51,54,56,59,60,63,65,68,70,72,77$, & \\
& $79,81,82,86,90$ & \\
\hline
\end{tabular}

PREGUNTA 3

\begin{tabular}{|c|c|}
\hline $\begin{array}{c}\text { No se puede determinar el } \\
\text { valor de la suma }\end{array}$ & $\begin{array}{c}\text { El resultado de la suma tiende a un número } \\
\text { finito (pero no llega a ese número) }\end{array}$ \\
\hline 42,75 & $\begin{array}{c}2,3,6,10,11,12,15,19,20,21,23,30,33,35,36,39,40,42,45,51, \\
52,57,59,61,62,63,67,71,75,79,86,88,90\end{array}$ \\
\hline
\end{tabular}

PREGUNTA 2

\begin{tabular}{|c|c|c|c|c|}
\hline \multicolumn{4}{|c|}{ Responde afirmativamente } \\
\hline $\begin{array}{c}\text { No calcula. } \\
\text { Afirma, se puede calcular un } \\
\text { aproximado, no exacto. }\end{array}$ & \multicolumn{3}{|c|}{ Indica una distancia } & \multirow{2}{*}{$\begin{array}{c}\text { Con una serie. } \\
\text { Valor aproximado }\end{array}$} \\
\cline { 2 - 4 } & $\cong 4$ & $\cong 6$ & $\rightarrow \mathrm{a} 2 \mathrm{~m}$ & 40,90 \\
\hline 20 & 51,67 & 4,85 & 1 & \\
\hline
\end{tabular}

Responde negativamente

\begin{tabular}{|c|c|}
\hline $\begin{array}{c}\text { No se podrá dar un número } \\
\text { exacto sino una aproximación }\end{array}$ & $\begin{array}{c}\text { Sería el límite de la serie } \\
\mathrm{h}+2 \mathrm{~h} / 2+2 \mathrm{~h} / 4+\ldots\end{array}$ \\
\hline $22,35,63$ & 9 \\
\hline
\end{tabular}

PREGUNTA 4

\begin{tabular}{|c|c|c|}
\hline La función tenderá a $0,2,1$ o al eje de las abcisas & $\begin{array}{c}\text { Se va convirtiendo } \\
\text { en una línea recta }\end{array}$ & $\begin{array}{c}\text { No se puede } \\
\text { determinar }\end{array}$ \\
\hline $\begin{array}{l}1,2,3,5,6,7,8,10,12,16,17,18,19,20,21,22,23,24,25,26,27,28,29,30,31,33,35,38,39,40, \\
44,45,47,48,50,51,53,54,55,56,57,59,60,62,63,69,70,71,76,77,79,84,85,86,87,88,90\end{array}$ & 42 & 13,89 \\
\hline
\end{tabular}

PREGUNTA 5

\begin{tabular}{|c|c|c|c|c|c|}
\hline \multicolumn{3}{|c|}{ Para ningún valor } & \multicolumn{3}{|c|}{ No se puede calcular $n$} \\
\hline La suma $\rightarrow 2$ & $\begin{array}{c}\text { La suma nunca } \\
\text { llegará a 2 }\end{array}$ & $\begin{array}{c}\text { Por el } \\
\text { ejercicio 2 }\end{array}$ & $\begin{array}{c}\text { La suma } \\
\text { se hace infinitesimal }\end{array}$ & $\begin{array}{c}\text { Un } n \text { grande } \\
\text { que no se puede calcular }\end{array}$ & $6,19,47,46$ \\
\hline $\begin{array}{c}2,3,13,23,28, \\
33,35,79\end{array}$ & $\begin{array}{c}4,12,30,39,40,50, \\
53,54,70,82,86\end{array}$ & 36 & 22 & 69 \\
\hline
\end{tabular}

